\title{
Micromechanical modelling of matrix cracks effect on shear and transverse response for unidirectional composites with a full field approach
}

\author{
B.Burgarella, A.Maurel-Pantel*, N.Lahellec, C.Hochard \\ Aix Marseille Univ, CNRS, Centrale Marseille, LMA, Marseille, France
}

\begin{abstract}
Modelling the damage of composite materials is not an easy task because different modes of ruins coexist: Fiber matrix decohesion,matrix cracks, delamination, and fiber cracks. In the case of laminated composites, the matrix cracks have the particularity to remain parallel to the fibers. As a consequence of the orientation of this crack network, only shear and transverse moduli in the plane of the ply are degraded in proportion to the increase of the crack density. The main point of this work is to characterize the relation linking transverse and shear damage with respect to the crack density. Following this objective, full field calculations are run using CraFT, a software developed at the LMA. The modeling is done in two steps: first the undamaged composite is homogenized, then, as a second step, the damaged behavior is determined by introducing cracks into the healthy composite. The behavior is calculated from an optimal size of RVE (Representative Volume Element) in order to determine numerically the relation between transverse and shear moduli variables.

Keywords: homogenization, microcracked composite, stiffness degradation, transverse damage
\end{abstract}

2010 MSC: 00-01, 99-00

\footnotetext{
* Corresponding author

Email address: maurel@lma.cnrs-mrs.fr (A.Maurel-Pantel)
} 


\section{Introduction}

Polymer-matrix composites (PMCs) have been increasingly used by industries due to their potential to fulfill the weight, mechanical and environmental requirements needed for structural applications ranging from aeronautic, automotive to sports sectors. Unfortunately the design of such structural parts, submitted overtime to complex loading, is not reliable enough to optimally use these materials. Nowadays a need to understand reliability and damage effect and evolution to improve design exists. The rupture of composite materials can be due to many mechanisms acting on various scale [1, 2, 3]. Two main damage mode are responsible of composite ruptures: fiber failure and matrix cracking. Failure in the fiber direction usually has catastrophic effects on both the laminate and the structure, the fracture propagates quasi-instantaneously through the thickness of the laminate thus leading to the complete rupture of the composite. This unstable propagation is due to the high level of loading required to break the ply in the fiber direction. When considering laminated composites with unidirectional plies of continuous fibers, whose strength and rigidity are very large compared to those of the polymer matrix, the cracks in the matrix have the particularity to remain parallel to the fibers regardless of the loading in the plane. As a consequence of the orientation of this crack network, only the shear and transverse moduli in the plane of a unidirectional ply are affected by the increase of crack density [4. For axial and transverse stiffness measurements, there are well-known experimental techniques in static and/or fatigue loading. These methods are widely used and many procedures can be found in ASTM standards [5]. However measurements of shear modulus for composite material is a more recent subject in literature. Standard methods exists: the Iosipescu shear test $\left[\underline{6}\right.$, the $[ \pm 45]_{S}$ coupon tension test [7], the Arcan test, and the torsion test. The torsion test seems to be the best method to measure the shear modulus, due to the geometry without free edge effect and uniform stress state $\left[8,9\right.$. Yet, the Iosipescu and the $[ \pm 45]_{S}$ coupon tension tests are very popular in composite characterization because the specimen are 
very easy to produce and test with a classic tensile machine. Salavatian et al. proposed a modified Iosipescu coupon to describe the evolution of damage due to shear and transverse loading and their interactions. Tests have been led on $\left[45 /-45 / 90_{6}\right]_{S}$ and $\left[0,90_{7}\right]_{S}$ S-glass epoxy laminates [10].

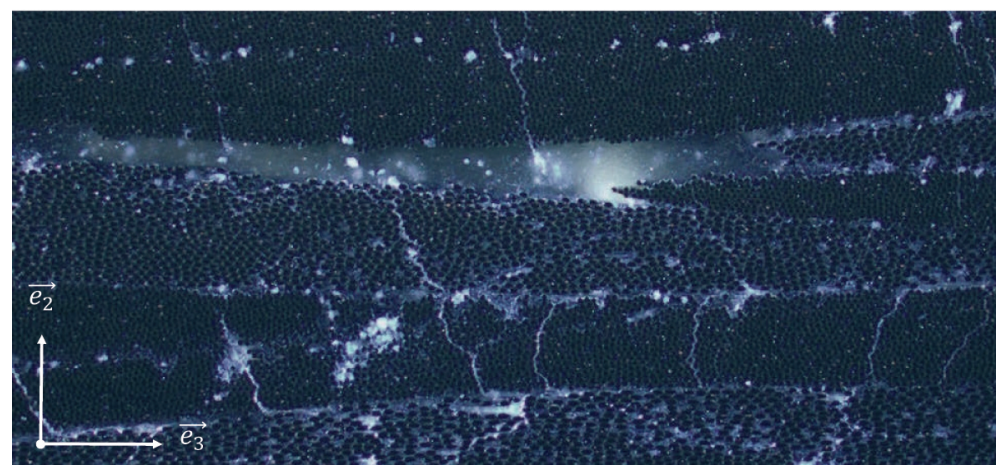

Figure 1: Cracks in a damaged composite observed with a microscope.

Figure 1 relates microscopic observations of cracks in unidirectional composite. Cracks are parallel to the fiber and propagate along the fiber direction. They generally pass through plies thickness keeping a certain width, but usually remain closed. This feature makes the cracks difficult to observe even with a micro tomography scanner. The damage induced gradually by matrix cracking (i.e. small cracks running parallel to the fiber direction [11]) can be described by phenomenological models based on continuum damage mechanics [12, analytical models [13, 14, 15], or numerical models [16]. Depending on the composite anisotropic degree, several damage variables can be used. In case of transverse isotropic material and plane stress assumption, three damage variables are considered:

$$
\left\{\begin{array}{c}
E_{1}^{d}=\left(1-d_{1}\right) E_{1}^{0} \\
E_{2}^{d}=\left(1-d_{2}\right) E_{2}^{0} \\
G_{12}^{d}=\left(1-d_{12}\right) G_{12}^{0}
\end{array}\right.
$$


Where $d_{1}$ corresponds to the fiber damage, it is a binary mechanisms related to the brittle fracture of composite. And $d_{2}$ et $d_{12}$ corresponds to matrix cracking. In literature both variables are supposed to be interrelated and a linear relation is classically defined between them [11, 9, 17]:

$$
d_{12}=c \times d_{2}
$$

Where $\mathrm{c}$ is the coupling coefficient between the transverse and the shear damage. Generally, the shear damage is empirically supposed to be proportional to the transverse damage and $c$ is equal to 1 [9].

The aim of this work consists in the determination of the relation between the transverse damage $d_{2}$ and the shear damage $d_{12}$ with a micromechanical approach based on full field method for unidirectional laminated composite. The paper proceeds first by the introduction of the sequential full field homogenization method, based on FFT calculation, and developed to calculate the stiffness of the healthy and damaged composite (Section 2). Then calculations are performed on RVEs (Representative Volume Elements) corresponding to different damage level (different initial void fraction and different ratio aspect of cracks). The damage values are then extrapolated for a representative cracks modelling 15 (Section 3). In Section 4, the predicted evolution of transverse and shear damage is observed and confronted with literature theoretical and experimental results. Finally, conclusions are drawn in Section 5.

\section{Calculation of Damaged Composite Elastic Properties}

In this section, a methodology is proposed to determine the elastic properties of an healthy composite $\left(\bar{C}_{0}\right)$ and a damaged composite $\left(\bar{C}_{d}\right)$ at different level of damage $d$ (i.e. different cracking rates) by means of full field homogenization calculations led on representative microstructure corresponding to the glass fiber reinforced epoxy composite. Calculation are performed using CraFT software 
[18, 19], that allows calculations on RVE using Fast Fourier Transform (FFT)

25 method (see Appendix A. The input data in CraFT are: a RVE in the form of a $3 \mathrm{D}$ or $2 \mathrm{D}$ picture, a behavior law for each material involved in the RVE, and a stress or strain loading. CraFT returns either the macroscopic strain or macrospic stress composite response, depending on the loading conditions and hence to the elastic properties of the composite.

30 2.1. Assumptions

For the composite considered in these investigations, epoxy matrix $(E=3$ $\mathrm{GPa}, \nu=0.33)$ and the glass fibers $(E=70 \mathrm{GPa}, \nu=0.22)$ are considered isotropic and purely elastic. The fiber volume fraction is fixed at $52 \%$. Consequently the macroscopic behavior of the healthy or damaged composite is considered orthotropic and purely elastic, and can be written with the following tensor expression [20]:

$$
\left[\begin{array}{c}
\left\langle\varepsilon_{11}\right\rangle \\
\left\langle\varepsilon_{22}\right\rangle \\
\left\langle\varepsilon_{33}\right\rangle \\
\left\langle\varepsilon_{23}\right\rangle \\
\left\langle\varepsilon_{13}\right\rangle \\
\left\langle\varepsilon_{12}\right\rangle
\end{array}\right]=\left[\begin{array}{cccccc}
\frac{1}{E_{1}} & \frac{-\nu_{21}}{E_{2}} & \frac{-\nu_{31}}{E_{3}} & 0 & 0 & 0 \\
\frac{-\nu_{12}}{E_{1}} & \frac{1}{E_{2}} & \frac{-\nu_{32}}{E_{3}} & 0 & 0 & 0 \\
\frac{-\nu_{13}}{E_{1}} & \frac{-\nu_{23}}{E_{2}} & \frac{1}{E_{3}} & 0 & 0 & 0 \\
0 & 0 & 0 & \frac{1}{2 G_{23}} & 0 & 0 \\
0 & 0 & 0 & 0 & \frac{1}{2 G_{13}} & 0 \\
0 & 0 & 0 & 0 & 0 & \frac{1}{2 G_{12}}
\end{array}\right]\left[\begin{array}{c}
\left\langle\sigma_{11}\right\rangle \\
\left\langle\sigma_{22}\right\rangle \\
\left\langle\sigma_{33}\right\rangle \\
\left\langle\sigma_{23}\right\rangle \\
\left\langle\sigma_{13}\right\rangle \\
\left\langle\sigma_{12}\right\rangle
\end{array}\right]
$$

Where $\langle f\rangle$ define the average of the field $f(x)$ on the RVE, as described in equation 4 .

$$
\langle f\rangle=\frac{1}{V} \int_{V} f d x
$$

The influence of cracking on the behavior of laminated composite is investigated under the plane stress assumption. The behavior law defined in equation 3 , is then reduced with the values of stress $\left(\left\langle\sigma_{13}\right\rangle=0,\left\langle\sigma_{23}\right\rangle=0,\left\langle\sigma_{33}\right\rangle=0\right)$ and considering the assumption that $E_{1}$ is directly linked to the fiber and stay independent of cracking rate. Therefore, the only the parameters that needs to be considered are the transverse modulus $E_{2}$ and the shear modulus $G_{12}$. 


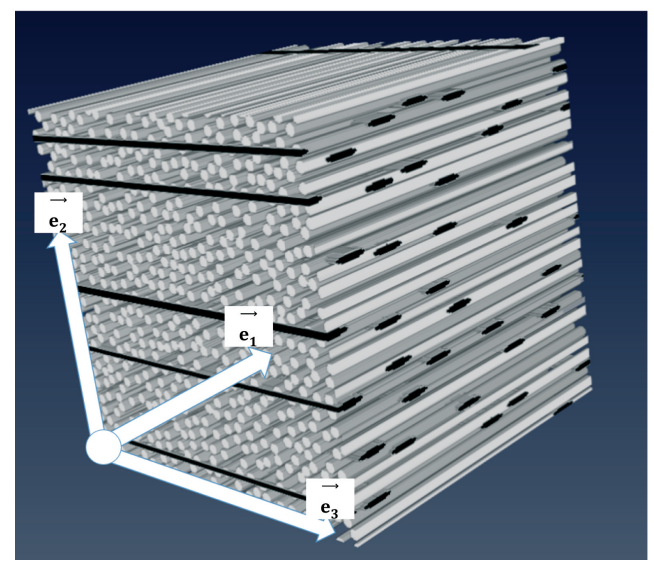

Figure 2: Numerically generated Representative Volume Element (RVE) in 3D of the damaged composite modelled with the three main axis $\left(\overrightarrow{e_{1}}, \overrightarrow{e_{2}}, \overrightarrow{e_{3}}\right)$. To improve visibility, cracks represented are not on scale.

\subsection{Cracks Modelling}

As it can be seen on the microscopic observation of a damaged laminated composite related in Figure 1. The damage consists of disseminated small cracks running parallel to the fibers, in the orthogonal direction to the fiber, they generally pass through the ply. These cracks are difficult to observe, because they are closed up and theirs shapes can be complex. The representative volume elements are scaled to the ply. They respect the direction of cracks observed and propose an idealized cracks shape. That is why in our RVE, cracks are modelled with an elliptic form, crossing the ply, with a minor axis equal to zero (due to the close up of the cracks) and a major axis as related in Figure2.

\subsection{Sequential Method of Homogenization}

The first step consist in the calculation of the elastic properties of the unidirectional composite thanks to the RVE illustrated in Figure 2. Running these calculations on such a 3D RVE require a lot of calculation time. So to solve this problem, the methodology proposed in this article consists in separating the homogenization process in two phases as already performed in literature [21, 22]: A first calculation is run on a 2D RVE taking into account the epoxy matrix 

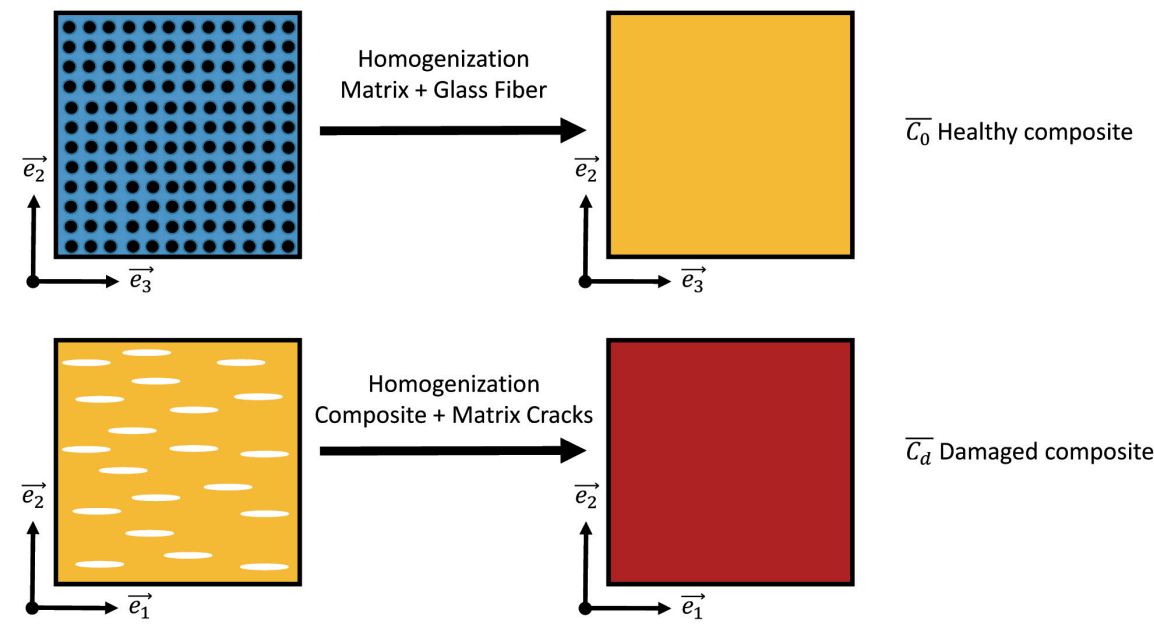

Figure 3: Synoptic of the homogenization sequential method used to calculate damaged composite elastic properties.

and the glass fibers, thus obtaining the orthotropic behavior of the healthy composite. Then a second calculation is run on another 2D REV taking into account the homogenized healthy composite behavior and the cracks. One 3D calculation has been replaced by two $2 \mathrm{D}$ calculations, but the first calculation is performed only once. The total calculation time is therefore drastically reduced. The synoptic of the proposed methodology is summed up in Figure 3 . The first calculation is performed on CraFT software and the elastic properties of the glass fiber reinforced healthy composite $\left(\bar{C}_{0}\right)$ are calculated and the following material constants are obtained:

$$
\left\{\begin{array}{c}
E_{1}=36479.2 \\
E_{2}=8606.44 \\
E_{3}=8664.37 \\
G_{23}=3168.71 \\
G_{13}=3478.1 \\
G_{12}=3473.43
\end{array}\right.
$$




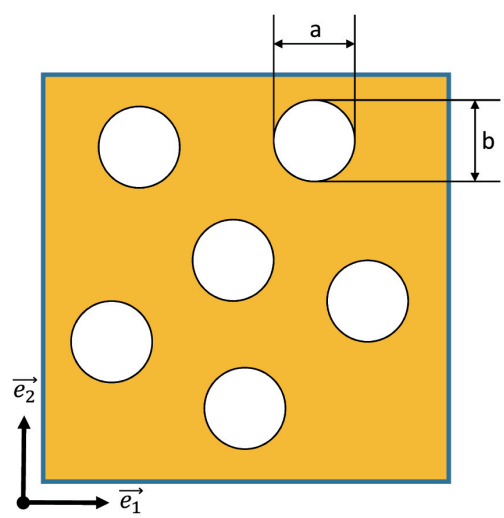

Initial void rate with a crack aspect ratio of 1

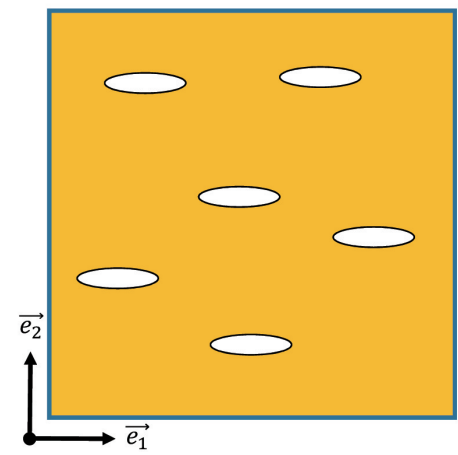

Initial void rate with a crack aspect ratio of 0,25

Figure 4: Numerically generated REV of damaged composite for a cracks aspect ratio of 1 and 0.25 describing the flattening of cracks along the minor axis of the ellipse.

\subsection{Damaged RVE}

The calculations are led on a numerically generated RVE, they need to represent different damage rates and have to take into account the ideal shape of cracks described in previous section. Moreover, CraFT is based on spectral numerical methods using a microstructure in image format, where each pixel represents one of the components of the composite material. In the considered case, the composite and cracks are present in the microstructure, if we want to describe accurately a crack in the RVE, it is necessary to keep a minimum number of pixels along the minor axis of the ellipse. Thats why, we defined a parameter $r$, named the cracks aspect ratio of the REV as related in the folowing equation:

$$
r=\frac{b}{a}
$$

Where $b$ and $a$ are respectively the minor and major axes of the ellipse. Calculations are performed for five aspect ratios. The lowest considered aspect ratio in the calculations is 0.01 .

Moreover, to investigate the damage evolution several cracking (or void) rates are considered, these rates are of course directly related to the damage rate. 


\begin{tabular}{|c|c|c|}
\hline $\begin{array}{c}\text { Cracking rates } \\
\text { for an aspect ratio } r=1 \\
\text { (or Initial void rates) }\end{array}$ & Cracks aspect ratio $r$ & $\begin{array}{c}\text { Crack density } d_{C} \\
\text { for the aspect ratio } r=0 \\
\left(\text { pixel }^{-1}\right)\end{array}$ \\
\hline $50 \%$ & $(1,0.5,0.2,0.1,0.01)$ & $1.6010^{-4}$ \\
\hline $40 \%$ & $(1,0.5,0.2,0.1,0.01)$ & $1.4410^{-4}$ \\
\hline $30 \%$ & $(1,0.5,0.2,0.1,0.01)$ & $1.2510^{-4}$ \\
\hline $20 \%$ & $(1,0.5,0.2,0.1,0.01)$ & $1.0610^{-4}$ \\
\hline $10 \%$ & $(1,0.5,0.2,0.1,0.01)$ & $7.5210^{-5}$ \\
\hline $5 \%$ & $(1,0.5,0.2,0.1,0.01)$ & $5.2810^{-5}$ \\
\hline $0.5 \%$ & $(1,0.5,0.2,0.1,0.01)$ & $1.6810^{-5}$ \\
\hline
\end{tabular}

Table 1: Cracking rates, aspect ratio used, and crack density to define the numerically generated REV.

Seven different cracking rates have been considered. For each cracking rate, the cracks are progressively flattened until the minimal ratio is reached. At the minimal ratio, where $r=0$ we choose to define a crack density with the following expression:

$$
d_{C}=\frac{N * a}{L^{2}}
$$

Where $d_{C}$ is the crack density, $\mathrm{N}$ the number of cracks introduced in RVE, a the length of the crack with an aspect ratio $r=0$, and $\mathrm{L}$ the resolution of the RVE (i.e number of pixel on a side). Overall, 35 RVEs are numerically generated and tested with CraFT. The cracking rates, the cracks aspect ratios and the crack density considered in this investigation are summed up in Table 1. Before discussing the results, a convergence analysis is essential to make sure that the RVEs are representatives. In order to achieve this, the RVE minimal resolution in pixel and the minimal cracks number necessary in the RVE have to be defined. 


\begin{tabular}{|c|c|c|c|c|c|c|}
\hline Pixels $L$ & 1000 & 2000 & 4000 & 6000 & 8000 & 10000 \\
\hline \hline$E_{2}$ & 7937 & 8032 & 8011 & 8045 & 8016 & 8011.455 \\
\hline$\Delta(\%)$ & 0.93 & 0.26 & 0.01 & 0.42 & 0.06 & 0.00 \\
\hline \hline$G_{12}$ & 3399 & 3402 & 3404 & 3404 & 3404 & 3405.3 \\
\hline$\Delta(\%)$ & 0.18 & 0.10 & 0.04 & 0.04 & 0.04 & 0.005 \\
\hline
\end{tabular}

Table 2: VER resolution convergence results.

\begin{tabular}{|c|c|c|c|c|}
\hline Cracks Number & $\mathbf{4}$ & $\mathbf{9}$ & $\mathbf{1 6}$ & $\mathbf{6 4}$ \\
\hline REV Size $L^{2}$ & $1000^{2}$ & $1500^{2}$ & $2000^{2}$ & $4000^{2}$ \\
\hline \hline$E_{2}$ & 2970 & 3767 & 3692 & 3655 \\
\hline$\Delta(\%)$ & 18.74 & 3.06 & 1.01 & 0.00 \\
\hline \hline$G_{12}$ & 2817 & 2893 & 2893 & 2894 \\
\hline$\Delta(\%)$ & 2.66 & 0.03 & 0.03 & 0.00 \\
\hline
\end{tabular}

Table 3: VER number of cracks convergence results.

60

\subsection{Convergence Analysis}

For the convergence analysis, we considered the worst RVE which is the one that includes both the smallest cracks aspect ratio and the highest cracking rate. The first step concerns the resolution sensitivity of the RVE, 6 resolutions are tested and the results, related in Table 2 , compare the transverse and shear moduli values. Then the cracks number sensitivity is investigated considering 4 different zooms on the cracked microstructure. Calculations are performed, and the values of transverse and shear modulus are compared as related in Table 3. In conclusion, the pixel resolution chosen for all the REVs is $10000^{2}$ pixels ( $L=10000)$ and the representative number of cracks is defined at 16 .

\section{Calculation on damaged RVE}

Calculations are performed on the $35 \mathrm{REVs}$ described in the previous section. The damaged composite elastic properties $\left(C_{d}\right)$ are obtained and used to calculate the value of the transverse damage $d_{2}$ and the shear damage $d_{12}$ 

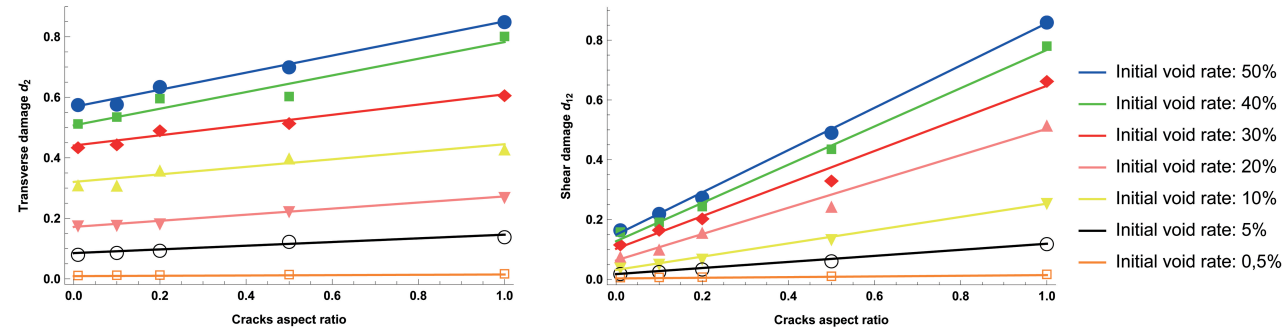

Figure 5: Evolution of transverse damage $d_{2}$ and the shear damage $d_{12}$ as a function of the initial void rate and the cracks aspect ratio calculated on numerically generated REVs.

damage in using the equation 1 . Results are reported in Figure 5. With the assumptions introduced previously, the value that we are trying to define, is the transverse and shear damage value corresponding to a cracks aspect ratio $r=0$ (i.e. when the cracks are closed up). To determine this value, the easiest way is to make a linear extrapolation of each cracking rates considered, and then extrapolate its limit at 0 as related in Figure 5. Then, with these extrapolated
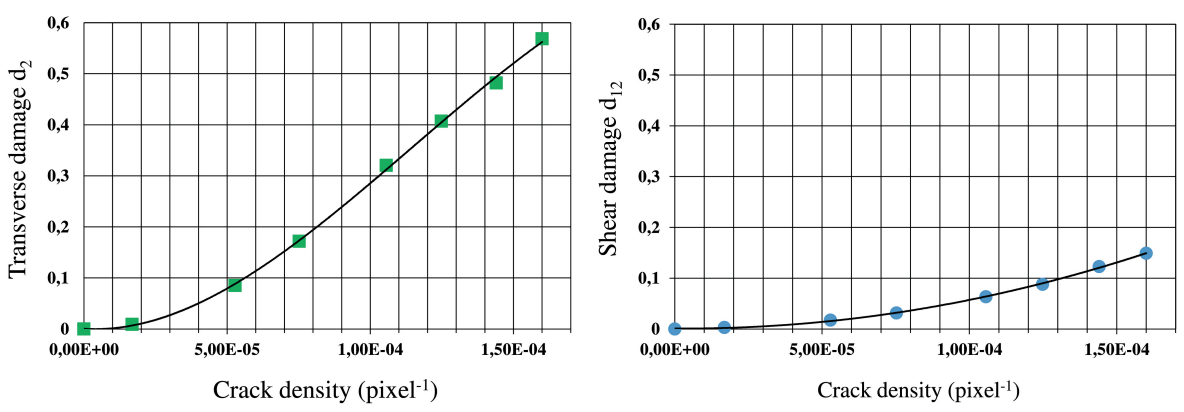

Figure 6: Evolution of the transverse damage and shear damage as a function of the crack density for a cracks aspect ratio $r=0$ as related in Table 1

values, the evolution of the transverse and shear damage as a function of the crack density related in Table 1 can be drawn in Figure 6. On the calculated evolution, the shear damage seems to present a nonlinear evolution and increase slower than the transverse damage. The transverse damage displays a nonlinear behavior at low values of crack density, and a linear evolution at high values of 85 crack density, increasing slowly at the beginning, then accelerating to reach a 
constant gradient value.

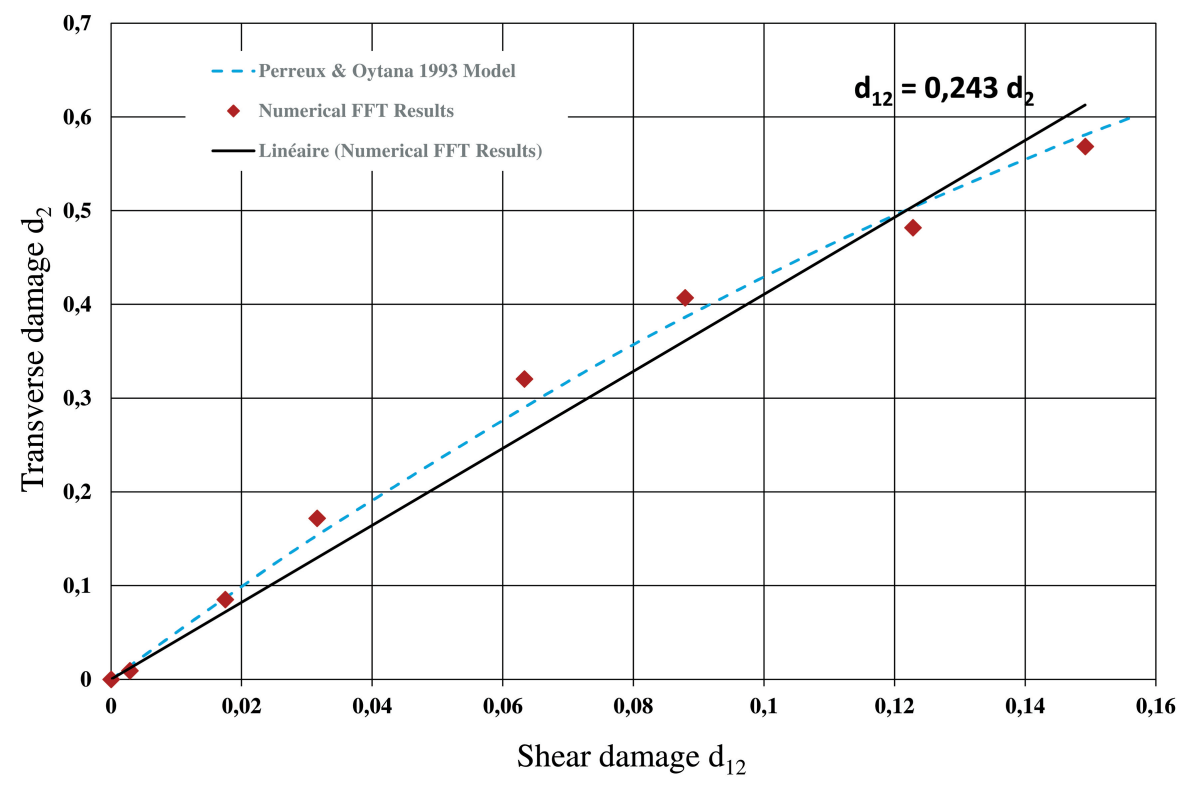

Figure 7: Transverse damage as a function of shear damage calculated for a cracks aspect ratio $r=0$ with the interpolation of the coupling coefficient $c$ value. Confrontation between Perreux and Oytana model [23] and our numerical FFT results.

\section{Results on transverse and shear damage ratio}

With these results, the evolution of the coupling coefficient between the transverse and the shear damage can be plotted for different damage rates in the unidirectional ply of the laminated composite considered. Figure 7 proposes to describe the evolution of the coupling coefficient $c$ for the 7 different cracking rates considered in the RVE numerically tested. The evolution of the coupling coefficient seems not to follow a totally linear behavior, but we chose to keep the linear relation related in equation 2 in the first section. On the Figure 7 a linear interpolation for the coupling between transverse and shear damages is proposed. After identification the coefficient $c$ is equal to 0.243 which is different from the value classically reported in literature. We then compare the 
numerical predictions with the model developed by Perreux and Oytana [23. The authors have proposed to model the problem in mean field homogenization framework with a self-consistent model on a microstructure. They modeled an unidirectional composite with microcracks parallel to fiber axis, after analytical calculation they obtained the following expression:

$$
d_{12}=1-\frac{1}{G_{12}}\left[\frac{1}{G_{12}}+\frac{d_{2}}{\sqrt{\left(1-d_{2}\right) E_{1} E_{2}}}\right]^{-1}
$$

A good correlation is observed between the numerical FFT results and the analytical model proposed by Perreux and Oytana [23]. But when we compare the linear interpolation and the analytical model prediction with the numerical FFT results, the respective errors are not so different. With the Figure 8 a con-

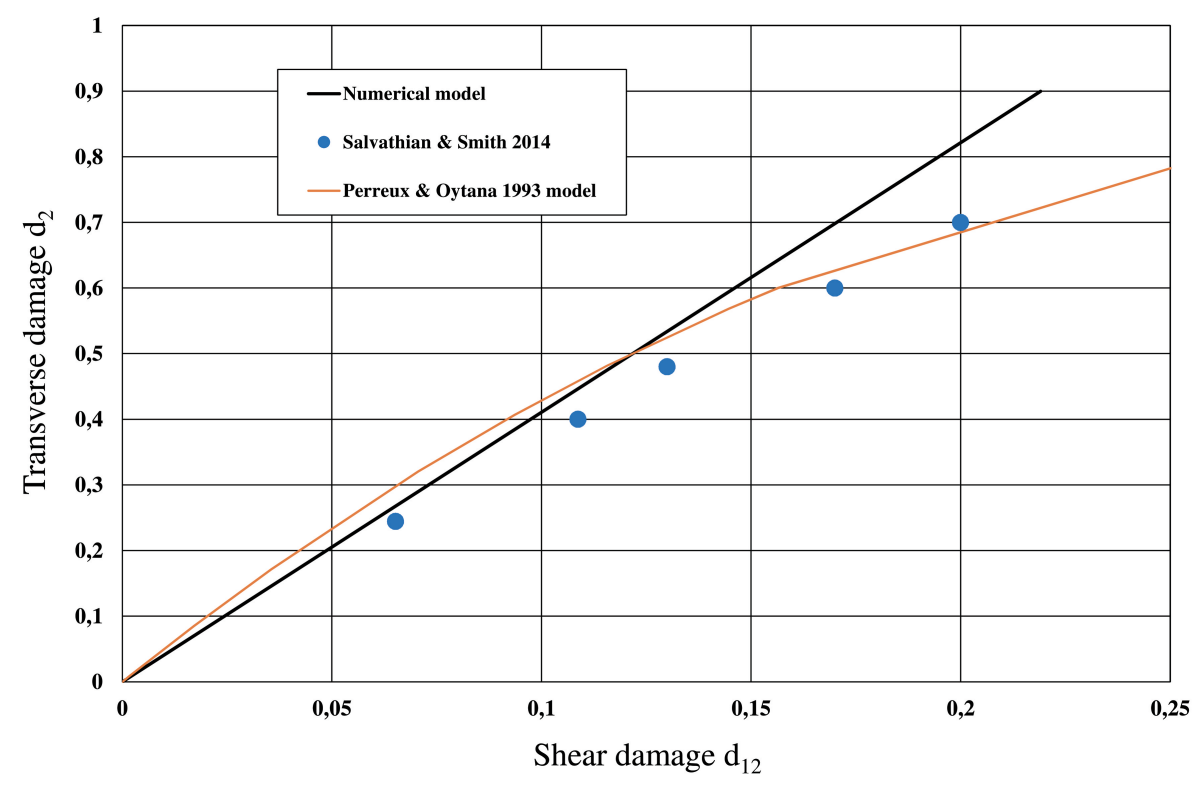

Figure 8: Confrontation between experimental results [10 available in literature on $\left[0,90_{7}\right]_{S}$ S-glass epoxy laminates Iosipescu specimens, the linear coupling coefficient c calculated and Perreux and Oytana model [23.

frontation is performed between the numerical law identified and experimental 
tests obtained recently by Salvathian and Smith on $\left[0,90_{7}\right]_{S}$ Iosipescu modified coupon in S-glass epoxy laminates [10]. A good correlation is observed between the experimental data and the coefficient identified with the micromechanical methodology proposed in this paper.

\section{Conclusions}

In this paper, a micromechanical approach based on a full field method is de-

$\mathrm{c}$ is difficult to experimentally identify, but is very useful in damage models to 
predict the ruining modes of laminated composite under static or fatigue loads. A prospect consists in developing new experimental tests or configurations to be able to observe the transverse and shear elastic properties for different damage levels avoiding early fracture in order to correlate our results on several composite materials (glass, carbon, or natural long fibers composite with thermoset or thermoplastic matrix). Generally this parameter is determined with an inverse method of identification or chosen equal to 1 . In this paper, with the developed methodology, it is possible to investigate the composite response at a specific cracking rate, but not to model the damage evolution in the ply. To do that, other methods should be investigated with meshless methods (XFEM, or fracture criteria 24) based on FEM simulations to describe the crack initiation and propagation in the ply or the laminate in order to have a better description of 135 the damage kinetic and improve the prediction of composite structure longevity. 


\title{
Appendix A. Homogenization method for periodic material
}

\author{
Appendix A.1. Problem to be solved
}

Homogenization is based on the distinction between two scales: the microscopic scale, corresponding to that of the microstructure, where each of the components constitutes a phase (fibre, matrix). At this scale, the microstructure is characterized by a representative elementary volume (RVE) $V$ of characteristic dimension 1 . The macroscopic scale, corresponding to the scale of the structure of characteristic dimension L. In the case of linear elasticity, when the assumption of separation of the scales is valid $(L>>l)$, the behaviour of the material constituting the structure can be replaced by the homogenized behaviour. This macroscopic behavior is defined by:

$$
\boldsymbol{\Sigma}=C^{h o m}: \mathbf{E}
$$

with $\boldsymbol{C}^{\text {hom }}$ the tensor of the effective elastic moduli, $\boldsymbol{\Sigma}$ and $\boldsymbol{E}$ the means of the stress fields $\sigma$ and strain fields $\varepsilon$ solution of the balance problem $P_{\text {micro }}$ given by:

$$
\left(P_{\text {micro }}\right) \begin{cases}\operatorname{div} \boldsymbol{\sigma}=0 & \text { in } V, \\ \boldsymbol{\sigma}(x)=\boldsymbol{C}^{(r)}: \boldsymbol{\varepsilon}(x) & \text { in phase }(r), \\ \varepsilon(x)=\nabla^{S}\left(u^{*}(x)\right)+\boldsymbol{E} & \text { in } V, \\ u^{*} \mathrm{~V}-\text { periodic and } \boldsymbol{\sigma} . n \mathrm{~V}-\text { antiperiodic } & \text { on } \partial V, \\ \left\langle\boldsymbol{\nabla}^{S}\left(u^{*}(x)\right)\right\rangle=0 . & \end{cases}
$$

with $\boldsymbol{C}^{(r)}$ the tensor of the elastic moduli in phase $(r)$ and $\boldsymbol{\nabla}^{S}\left(u^{*}(x)\right)$ the fluctuating part of the strain tensor.

Appendix A.2. The FFT Method

In order to solve numerically the equations A.2, a method based on Fast Fourier Transforms (FFT) has been developped at the LMA by H. Moulinec and P. Suquet [18, 19]. This method has been implemented in a software named CraFT (Composite Response and Fourier Transforms) available under Cecil B 
licence on the LMA website (http://craft.lma.cnrs-mrs.fr/). The problem A.2 is replaced by the auxiliary problem A.3 involving an homogeneous linear elastic body defined by its stiffness $\boldsymbol{C}^{0}$ and under a polarization field $\boldsymbol{\tau}(x)$. This problem can be expressed as:

$$
\left(P_{\text {micro }}\right) \begin{cases}\operatorname{div} \boldsymbol{\sigma}=0 & \text { in } V, \\ \boldsymbol{\sigma}(x)=\boldsymbol{C}^{(0)}: \boldsymbol{\varepsilon}(x)+\boldsymbol{\tau}(x) & \text { in } V, \\ \boldsymbol{\tau}(x)=\left(\boldsymbol{C}^{(r)}-\boldsymbol{C}^{(0)}\right): \boldsymbol{\varepsilon}(x) & \text { in phase }(r), \\ \boldsymbol{\varepsilon}(x)=\boldsymbol{\nabla}^{S}\left(u^{*}(x)\right)+\boldsymbol{E} & \text { in } V, \\ u^{*} \mathrm{~V}-\text { periodic and } \boldsymbol{\sigma} \cdot n \mathrm{~V} \text { - antiperiodic } & \text { on } \partial V, \\ \left\langle\boldsymbol{\nabla}^{S}\left(u^{*}(x)\right)\right\rangle=0 . & \end{cases}
$$

The solution of this problem can be expressed thanks to the periodic Green operator $\boldsymbol{\Gamma}^{0}$ with the form:

$$
\varepsilon(x)=E-\Gamma^{0} * \tau(x) \quad \forall x \in V
$$

with $*$ expression the convolution product. Knowing that $\boldsymbol{\sigma}$ is an equilibrated field, this expression can be rewrite :

$$
\varepsilon(x)=\varepsilon(x)-\Gamma^{0} * \boldsymbol{\sigma}(x) \quad \forall x \in V
$$

In Fourier space, this solution is easier to compute since the convolution product becomes a tensorial product and the operator $\hat{\boldsymbol{\Gamma}}^{0}$ can be written in closed form. For an isotropic reference material with lame coefficients $\lambda^{0}$ and $\mu^{0}, \hat{\boldsymbol{\Gamma}}^{0}$ takes the form:

${\hat{\boldsymbol{\Gamma}^{0}}}_{i j k h}=\frac{1}{4 \mu^{0}|\xi|^{2}}\left(\delta_{k i} \xi_{h} \xi_{j}+\delta_{h} i \xi_{k} \xi_{j}+\delta_{k j} \xi_{h} \xi_{i}+\delta_{h j} \xi_{k} \xi_{i}\right)-\frac{\lambda^{0}+\mu^{0}}{\mu^{0}\left(\lambda^{0}+2 \mu^{0}\right)} \frac{\xi_{i} \xi_{j} \xi_{k} \xi_{h}}{|\xi|^{4}}$

This problem is then solved using the following iterative algorithm as can be seen in:

Initialization : $\boldsymbol{\varepsilon}^{0}(x)=\boldsymbol{E}, \quad \boldsymbol{\sigma}^{0}(x)=\boldsymbol{C}(x): \varepsilon^{0}(x) \quad \forall x \in V$ 
$\varepsilon^{i}$ and $\boldsymbol{\sigma}^{i}$ are known, repeat until convergence:

$$
\begin{array}{ccc}
\text { Real space } & & \text { Fourier space } \\
\boldsymbol{\sigma}^{i} & \text { FFT } \rightarrow & \hat{\boldsymbol{\sigma}}^{i} \\
& & \hat{\boldsymbol{\varepsilon}}^{i+1}(\xi)=\hat{\boldsymbol{\varepsilon}}^{i}-\hat{\boldsymbol{\Gamma}}^{0}(\xi): \hat{\boldsymbol{\sigma}}^{i}(\xi) \\
& & \hat{\boldsymbol{\varepsilon}}^{i+1}(0)=\boldsymbol{E} \\
\boldsymbol{\varepsilon}^{i+1}(x) & \leftarrow \mathrm{FFT}^{-1} & \hat{\boldsymbol{\varepsilon}}^{i+1}(\xi) \\
\boldsymbol{\sigma}^{i+1}(x)=\boldsymbol{C}^{(r)}: \boldsymbol{\varepsilon}(x) & &
\end{array}
$$

\section{References}

n [3] P. Maimi, P. Camanho, J. Mayugo, C. Davila, A continuum damage

a model for composite laminates: Part ii. computational implementa-

160

口

[1] A. S. D. Wang, Strength, failure and fatigue analysis of laminates, ASM International, 1987.

2] P. Maimi, P. Camanho, J. Mayugo, C. Davila, A continuum damage model for composite laminates: Part i. constitutive model, Mechanics of Materials 39 (10) (2007) 897 - 908. doi:https:

//doi.org/10.1016/j.mechmat.2007.03.005.

URL http://www.sciencedirect.com/science/article/pii/ S0167663607000543

tion and validation, Mechanics of Materials 39 (10) (2007) 909 - 919. doi:https://doi.org/10.1016/j.mechmat.2007.03.006

URL http://www.sciencedirect.com/science/article/pii/ S0167663607000555

q [4] A. Turon, P. Camanho, J. Costa, C. Davila, A damage model for the 165 s simulation of delamination in advanced composites under variablemode loading, Mechanics of Materials 38 (11) (2006) 1072 - 1089. doi:https://doi.org/10.1016/j.mechmat.2005.10.003

a URL http://www.sciencedirect.com/science/article/pii/ S0167663605002000 
[5] Test method for tensile properties of polymer matrix composite materials. doi:10.1520/d3039_d3039m-17.

[6] Test method for shear properties of composite materials by the v-notched beam method. doi:10.1520/d5379_d5379m-12.

[7] Test method for in-plane shear response of polymer matrix composite materials by tensile test of a 45 laminate. doi:10.1520/d3518_d3518m.

[8] M. Knops, C. Bgle, Gradual failure in fibre/polymer laminates, Composites Science and Technology 66 (5) (2006) 616-625. doi:10.1016/j. compscitech.2005.07.044.

[9] C. Hochard, Y. Thollon, A generalized damage model for woven ply laminates under static and fatigue loading conditions, International Journal of Fatigue 32 (1) (2010) 158-165. doi:10.1016/j.ijfatigue.2009.02.016

[10] M. Salavatian, L. Smith, The effect of transverse damage on the shear response of fiber reinforced laminates, Composites Science and Technology 95 (2014) 44-49. doi:10.1016/j.compscitech.2014.02.012

[11] P. Ladevèze, G. Lubineau, On a damage mesomodel for laminates: micromechanics basis and improvement, Mechanics of Materials 35 (8) (2003) 763-775. doi:10.1016/s0167-6636(02)00204-1.

[12] P. Ladevze, E. Ledantec, Damage modelling of the elementary ply for laminated composites, Composites Science and Technology 43 (3) (1992) 257267. doi:10.1016/0266-3538(92)90097-m.

[13] Z. Hashin, Analysis of cracked laminates: a variational approach, Me-

口. chanics of Materials 4 (2) (1985) 121-136. doi:10.1016/0167-6636(85) 90011-0

[14] S. Tan, R. Nuismer, A theory for progressive matrix cracking in composite 195 laminates, Journal of Composite Materials 23 (10) (1989) 1029-1047. doi: 10.1177/002199838902301006. 
[15] R. Nuismer, S. Tan, Constitutive relations of a cracked composite lamina, Journal of Composite Materials 22 (4) (1988) 306-321. doi:10.1177/ 002199838802200401

[16] J. M. Berthelot, Analysis of the transverse cracking of cross-ply laminates: A generalized approach, Journal of Composite Materials 31 (18) (1997) 1780-1805. doi:10.1177/002199839703101801.

[17] Y. Thollon, C. Hochard, A general damage model for woven fabric composite laminates up to first failure, Mechanics of Materials 41 (7) (2009) 820-827. doi:10.1016/j.mechmat.2009.02.009.

[18] H. Moulinec, P. Suquet, A fast numerical method for computing the linear and nonlinear properties of composites, Comptes-rendus de lAcadeemie des sciences serie II (318) (1994) 14171423.

[19] H. Moulinec, P. Suquet, A numerical method for computing the overall response of nonlinear composites with complex microstructure, Computer Methods in Applied Mechanics and Engineering 157 (1-2) (1998) 69-94. doi:10.1016/s0045-7825(97)00218-1.

[20] J. Lemaitre, J. Chaboche, A. Benallal, R. Desmorat, Mécanique des matériaux solides - 3ème édition, Physique, Dunod, 2009. URL https://books .google.fr/books?id=6kwLkhEc8cYC

[21] Y. Wu, R. Shivpuri, L. J. Lee, Effect of macro and micro voids on elastic properties of polymer composites, Journal of Reinforced Plastics and Composites 17 (15) (1998) 1391-1402. doi:10.1177/073168449801701507.

[22] I. Doghri, L. Tinel, Micromechanical modeling and computation of elastoplastic materials reinforced with distributed-orientation fibers, Interna-

a tional Journal of Plasticity 21 (10) (2005) 1919-1940. doi:10.1016/j. ijplas.2004.09.003 
[23] D. Perreux, C. Oytana, Continuum damage mechanics for microcracked composites, Composites Engineering 3 (2) (1993) 115-122. doi:10.1016/ $225 \quad 0961-9526(93) 90036-j$.

[24] D. Leguillon, Strength or toughness? a criterion for crack onset at a notch, European Journal of Mechanics - A/Solids 21 (1) (2002) 61-72. doi:10. 1016/s0997-7538(01)01184-6. 